\title{
Perspektivenübernahme als Kompetenz für den Geschichtsunterricht
}

\author{
Theoretische und empirische Zusammenhänge zwischen \\ fachspezifischen und sozial-kognitiven Schülermerkmalen
}

\author{
Ulrike Hartmann • Michael Sauer • Marcus Hasselhorn
}

Zusammenfassung: In dieser Studie wird eine Teilkompetenz historischen Denkens - die historische Perspektivenübernahme - bei Schülern untersucht. Zunächst wird die Kompetenz sowohl aus sozial-kognitiver als auch aus fachdidaktischer Perspektive vorgestellt. Weiterhin werden Befunde zum Bedingungsgefüge zwischen Schulleistung, Selbstkonzept und Interesse am Fach Geschichte dargestellt und mit der Kompetenz zur historischen Perspektivenübernahme verknüpft. Es werden Fragen nach den Beziehungen zwischen diesen fachspezifischen Leistungs- und Motivationsindikatoren, der Teilkompetenz zur historischen Perspektivenübernahme und der sozial-kognitiven Fähigkeit zur sozialen Perspektivenübernahme aufgeworfen. An 375 Gymnasiasten aus den Klassenstufen 7 und 10 werden diese Beziehungen in einem Querschnittsdesign untersucht. Die Ergebnisse zeigen, dass die Kompetenz historischer Perspektivenübernahme in der 7. Klasse mit der Fähigkeit zur sozialen Perspektivenübernahme korreliert, während in der 10. Klasse Korrelationen mit den fachspezifischen Variablen Geschichtsinteresse, Selbstkonzept in Geschichte, der Geschichtsnote und dem Ergebnis eines themenspezifischen Lückentextes bestehen. Ausgehend von diesen Befunden werden Herausforderungen für die Erfassung von Schülerkompetenzen in Geschichte insbesondere im Kontext von Bildungsstandards erörtert. Diese betreffen Reliabilitätsund Validitätsprobleme, die Kontextgebundenheit der Messung historischer Kompetenzen, die eingesetzten Aufgabenformate sowie die Formulierung von Stufenmodellen.

Schlüsselwörter: Bildungsstandards · Perspektivenübernahme $\cdot$ Geschichtsunterricht · historisches Denken

\section{Perspective taking as a competence for history education - Theoretical and empirical relations between subject-specific and socio-cognitive characteristics in students}

\footnotetext{
(C) VS-Verlag 2009
}

Dr. U. Hartmann $(\bowtie) \cdot$ Prof. Dr. M. Hasselhorn Deutsches Institut für Internationale Pädagogische Forschung, AE: Bildung und Entwicklung Schloßstr. 29, 60486 Frankfurt a.M., Deutschland

E-Mail: u.hartmann@dipf.de; hasselhorn@dipf.de

Prof. Dr. M. Sauer

Seminar für Didaktik der Geschichte, Georg-August-Universität Göttingen

Waldweg 26, 37073 Göttingen, Deutschland

E-Mail: msauer1@gwdg.de 


\begin{abstract}
This study addresses a competency of students' historical thinking related to taking perspectives. We start by discussing socio-cognitive theories from psychology as well as approaches from history education that focus on this competency. We also present empirical findings concerning relationships between achievement, self-concept and interest in the subject of history and connect these findings with the competency to take historical perspectives. Our research questions target this relationship between indicators of achievement and motivation in the subject of history, the competency of historical perspective taking and students' socio-cognitive ability to adopt social perspectives in their everyday lives. These questions are investigated using a crosssectional design with 375 grammar school students in grades 7 and 10. Results indicate that in grade 7 the competency to take historical perspectives relates to students' ability to coordinate social perspectives in their everyday lives. For 10th graders, however, the adoption of historical perspectives is closely related to subject-specific variables such as interest for history, self-concept, history grade and achievement in a test of historical knowledge. In the last section of this paper, we discuss challenges that arise when students' competencies in a subject like history are assessed within the context of standard-based testing. Specifically, we raise the issues of reliability, validity, the context-specificity of measurements, the kinds of response formats used and the formulation of progression models of historical thinking.
\end{abstract}

Keywords: Historical thinking $\cdot$ History education $\cdot$ Perspective taking $\cdot$ Standards for education

\title{
1 Einleitung
}

Seit Beginn der Debatte um Bildungsstandards (vgl. Klieme et al. 2003) sind zahlreiche Bestrebungen festzustellen, fachspezifische Kompetenzen für alle Schulfächer zu formulieren. Kompetenzen werden nach Weinert (vgl. 2001) als kognitive Fähigkeiten und Fertigkeiten zur Lösung von Problemen bezeichnet, verbunden mit motivationalen, volitionalen und sozialen Bereitschaften, diese in variablen Situationen zu nutzen. Ausgehend von dieser Definition wurde damit begonnen, vor allem im Bereich der Mathematik, des Lesens, der Naturwissenschaften und der Fremdsprachen Kompetenzmodelle zu formulieren, empirisch erfassbar zu machen und in konkretes Unterrichtsgeschehen umzusetzen. ${ }^{1}$

Da die Kompetenzdebatte die gesamte Schul- und Bildungslandschaft betrifft, sind die sogenannten „weicheren“ gesellschafts- und geisteswissenschaftlichen Schulfächer ebenfalls angesprochen und stehen vor einer umfassenden neuen Herausforderung (vgl. z.B. Tenorth 2008): denn eine Tradition, Schulleistungen und Kompetenzen zum Beispiel im Fach Geschichte systematisch zu erfassen, besteht bisher nicht. Vielfältig sind die Schwierigkeiten, wenn es darum geht, die heuristischen Ziele historisches Denken (vgl. z.B. Seixas 1996; Wineburg 1999) oder Geschichtsbewusstsein (vgl. z.B. Pandel 1987; von Borries 1995) in einzelne Teilkompetenzen aufzuschlüsseln, diese von rein deklarativem Faktenwissen abzugrenzen und messbar zu machen. Dennoch haben in den letzten Jahren die wissenschaftlichen Bemühungen zugenommen, Kompetenzmodelle historischen Denkens zu formulieren. Im internationalen Raum gehen solche Modelle bereits in bildungspolitische Anforderungskataloge ein (vgl. z. B. die National Standards for History in den USA; National Center for History in the Schools 1996). In Deutschland existieren momentan mehrere Kompetenzmodelle historischen Lernens nebeneinander. So wurde mit dem Modell der Gruppe FUER Geschichtsbewusstsein (vgl. z. B. Körber, 
Schreiber \& Schöner 2007; Schreiber 1999, 2008) eine Konzeption vorgeschlagen, die historische Frage, Orientierungs- und Methodenkompetenzen als konstituierend für historisches Denken ansieht. Pandel (2005, S. 27 ff.) schlägt ein Modell mit den Bestandteilen Gattungskompetenz, Interpretationskompetenz, narrative Kompetenz und geschichtskulturelle Kompetenz vor. Sauer (vgl. 2002, 2006) trennt dagegen Sachkompetenz, Deutungsund Reflexionskompetenz und Medien/Methodenkompetenz voneinander, die wiederum in verschiedene Teilkompetenzen unterteilt werden. Sauers Annahmen stellen zudem die Grundlage für die Bildungsstandards des Verbandes der Geschichtslehrer Deutschlands (vgl. 2006) dar. Bisher bleiben diese Ansätze weitgehend normativ, es liegen allenfalls erste empirische Befunde über die Ausprägungen von Schülerkompetenzen in den vorgeschlagenen Teilbereichen vor (vgl. z. B. Bernhardt 2007; Gautschi et al. 2007; Hartmann \& Hasselhorn 2008; Martens 2008).

Der Fokus der vorliegenden Studie liegt auf einer Teilkompetenz, die Bestandteil von geschichtsdidaktischen Modellen ist und damit als ein wichtiges Ziel historischen Lernens angesehen wird (vgl. z. B. Körber, Schreiber \& Schöner 2007; National Center for History in the Schools 1996; Sauer 2002, 2006). Es handelt sich dabei um die Fähigkeit von Schülern, sich in historische Akteure und Situationen hineinzuversetzen und dabei die Lebensbedingungen sowie die Denk- und Handlungshorizonte der jeweiligen Zeit zu berücksichtigen. Diese Kompetenz umschreiben wir mit dem Begriff historische Perspektivenübernahme, womit die theoretische Anlehnung an das entwicklungspsychologisch begründete und erziehungswissenschaftlich relevante Konstrukt der sozialen Perspektivenübernahme angedeutet wird. In der Literatur wird historischer Perspektivenübernahme neben der Bedeutung für Leistungen im Geschichtsunterricht auch eine Rolle für sozial kompetentes Handeln in der Gegenwart zugeschrieben (vgl. z. B. Ashby \& Lee 1987). Es existieren jedoch bisher keine empirischen Befunde zum Zusammenspiel historischer Perspektivenübernahme mit sozial-kognitiven und fachspezifischen Schülermerkmalen. Die vorliegende Studie liefert erste empirisch gestützte Hinweise zu dieser Schülerkompetenz für den Geschichtsunterricht.

\subsection{Perspektivenübernahme als Bindeglied zwischen sozialer Kompetenz und historischem Verstehen}

Nach Johnsons (vgl. 1975) Definition ist soziale Perspektivenübernahme die Fähigkeit zu verstehen, wie eine Situation sich für eine andere Person darstellt und wie diese Person kognitiv und emotional darauf reagiert. Die Zielperson ist dabei meistens ein Interaktionspartner, dessen Perspektive es zu verstehen gilt, und Perspektivenübernahme findet mit dem Ziel statt, Kommunikation zu verbessern und soziale Konflikte zu lösen (vgl. Selman 1980, 2003; Yeates \& Selman 1989). Soziale Perspektivenübernahme wird damit als ein integraler Bestandteil sozialer Kompetenz betrachtet (vgl. Caldarella \& Merell 1997; Jerusalem \& Klein-Heßling 2002; Selman 2003). Definitionen von Perspektivenübernahme im Kontext des Faches Geschichte setzen einen anderen Schwerpunkt. Lee und Ashby (vgl. 2001) ${ }^{2}$ verstehen das Konzept als ein Ergebnis einer historischen Rekonstruktionsleistung. Ihre Definition umfasst sowohl das Wissen, dass historische Akteure eine spezifische Perspektive auf ihre Welt hatten als auch das Verständnis dafür, wie diese Perspektive die Handlungen dieser Akteure in bestimmten Situationen beeinflusst hat. 
Historische Perspektivenübernahme bedeutet also, die Handlungsergebnisse, die dem Historiker (oder dem Schüler im Geschichtsunterricht) beispielsweise durch Text- und Bildquellen zugänglich gemacht werden, historisch plausibel aus Intentionen, Motiven und Emotionen der Akteure abzuleiten und zu erklären. Dabei ist die Erkenntnis entscheidend, dass die Gedanken und das Verhalten historischer Akteure maßgeblich von Normen und Wertvorstellungen der jeweiligen Zeit und Gesellschaft mitbestimmt wurden. Bergmann (vgl. 2000) beschreibt die Entwicklung von historischer Perspektivenübernahme bei Schülern als längeren Prozess, in dem zunächst eigene Erfahrungen und Lebensumstände auf frühere Zeiten übertragen werden. Nach und nach sind Schüler in der Lage, das Denken und Handeln früherer Menschen nachzuvollziehen und dabei auch frühere Lebensumstände einzubeziehen. Am Schluss dieses Prozesses können sie wahrnehmen, dass Menschen in ähnlichen Lebenslagen Unterschiedliches erreichen wollten und sich aufgrund ihrer Intentionen und Motive unterschiedlich verhalten haben. Historische Perspektivenübernahme entwickelt sich somit von einer gegenwartsfixierten Betrachtung historischer Situationen bis hin zur Berücksichtigung des historischen Kontextes und der dezidierten Wahrnehmung von Unterschieden zwischen Vergangenheit und Gegenwart. Downey (zit. nach Yeager \& Foster 2001, S. 17) nimmt an, dass historische Perspektivenübernahme dann vorliegt, wenn Schüler realisieren, dass die Vergangenheit sich von der Gegenwart unterscheidet und dass historische Handlungsergebnisse aufgrund spezifischer zeitlicher und räumlicher Bedingungen zustande kamen.

Diese unterschiedlichen Zwecke von Perspektivenübernahme - auf der einen Seite das sozial kompetente Handeln, auf der anderen Seite die adäquate Rekonstruktion historischer Wahrnehmungs, Denk- und Handlungshorizonte - führen jedoch nicht zu einer Unvereinbarkeit dieser beiden Konzepte. Ashby und Lee (vgl. 1987) vermuten, dass das Einnehmen historischer Perspektiven ebenfalls die Bewältigung von Herausforderungen in der Gegenwart erleichtert. Gehlbach (vgl. 2004a) nimmt an, dass die Fähigkeit zur Einnahme sozialer Perspektiven in alltäglichen Situationen ebenfalls die Fähigkeit begünstigen kann, sich in historische Akteure hineinzuversetzen. In einer Studie mit Neunt- und Zehntklässlern findet er moderate signifikante Zusammenhänge zwischen Maßen sozialer und historischer Perspektivenübernahme. Von Borries (vgl. 1995) vermutet, dass ein Mangel an historischer Perspektivenübernahme das Risiko birgt, auch in der Gegenwart nicht erkennen zu können, wenn beispielsweise die Gefahr zum Mitläufertum besteht. Schüler gäben sich damit einer „Überlegenheitsillusion“ (ebd., S. 388) gegenüber der Vergangenheit hin.

Einen Interventionsansatz, der historische Inhalte mit der Förderung von sozial kompetentem und gesellschaftlich verantwortungsvollem Handeln verknüpft, stellt das Unterrichtsprogramm „Facing History and Ourselves“ (FHAO) dar. In diesem Programm, welches vor allem an Schulen in den USA Bestandteil des Geschichts- oder Social Studies-Unterrichts ist, werden über die Beschäftigung mit Themen wie dem Holocaust Intergruppenkonflikte, moralische und ethische Fragen sowie Aspekte sozialer Gerechtigkeit und verantwortungsvollen Handelns in den Unterricht integriert. Die Förderung von sozialer Perspektivenübernahme ist ebenfalls zentraler Bestandteil dieses Programms. Eine Studie mit Achtklässlern, die an einem FHAO-Kurs teilnahmen, ergab Verbesserungen in der Koordination verschiedener Perspektiven bei gleichzeitiger Abnahme ras- 
sistischer Einstellungen sowie des selbstberichteten aggressiven Verhaltens (vgl. Hickey Schultz, Barr \& Selman 2001).

\subsection{Zusammenhänge zwischen historischer Perspektivenübernahme, Motivation und Leistung im Geschichtsunterricht}

Neben der Relevanz historischer Perspektivenübernahme für sozial kompetentes Verhalten von Schülern weist diese Kompetenz einen klaren fachspezifischen Bezug auf, wie es in der Expertise zu den Bildungsstandards (vgl. Klieme et al. 2003) gefordert ist. In der geschichtsdidaktischen Forschung wird Perspektivenübernahme als wichtiger Bestandteil des Unterrichtsfaches angesehen, erkennbar an zahlreichen Beiträgen (vgl. z. B. Baring 2004; Davis Jr., Yeager \& Foster 2001; Portal 1987; von Borries 1995). Es ist jedoch bisher unklar, ob sich diese theoretisch vermutete Relevanz für das Fach auch empirisch zeigen lässt, d.h., ob historische Perspektivenübernahme beispielsweise mit der Leistung oder dem Interesse für Geschichte zusammenhängt. Auch zu den Zusammenhängen von Geschichtsleistung und Geschichtsinteresse untereinander existieren nur vereinzelte Beiträge. Hierzu lohnt sich der Blick auf andere Schulfächer, die bereits intensiver erforscht wurden. Beispielsweise finden Denissen, Zarrett \& Eccles (vgl. 2007) in ihrer Längsschnittstudie positive (und mit dem Alter in ihrer Stärke zunehmende) Zusammenhänge zwischen Leistung, Selbstkonzept und Interesse in den Bereichen Englisch, Mathematik und Naturwissenschaften. Diese scheinen auch im Fach Geschichte zu existieren (vgl. Rost \& Sparfeldt 2002 für das Selbstkonzept sowie Kölbl, Tiedemann \& BillmannMahecha 2006 für das Interesse). Es liegen jedoch ebenfalls Studien vor, die dezidiert auf die Unterschiedlichkeit der Fächer hinweisen. So berichten Stodolsky, Salk und Glaessner (vgl. 1991) von deutlichen Unterschieden in Schülervorstellungen über Mathematik und Social Studies und Wolters und Pintrich (vgl. 1998) verweisen auf unterschiedliche Motivationslagen in Mathematik, Englisch und Social Studies.

Bei der Erforschung des Schulfachs Geschichte stehen insbesondere motivationale Komponenten wie das fachspezifische Interesse im Fokus. Gehlbach (vgl. 2006) weist auf die Notwendigkeit hin, das Interesse am Geschichtsunterricht besonders zu fördern. Perspektivenübernahme könnte dazu ein Mittel sein, da das Hineinversetzen in andere Zeiten und Situationen diese etwas näher an die Lebenswelt der Schüler heranbringen könnte und die oftmals riesig erscheinende Kluft zwischen Vergangenheit und Gegenwart verringern könnte. Portal (vgl. 1987) vermutet ebenfalls, dass die Kompetenz, historische Perspektiven einzunehmen, das Geschichtsinteresse von Schülern fördert. Kölbl, Tiedemann und Billmann-Mahecha (vgl. 2006) stellten in ihrer Untersuchung mit Viertklässlern einen deutlichen Zusammenhang zwischen dem Interesse und der Leistung im Fach Geschichte fest. Empirische Studien über den Zusammenhang zwischen Teilkompetenzen historischen Lernens und dem Geschichtsinteresse existieren bisher jedoch nicht.

Zum Zusammenhang zwischen historischer Perspektivenübernahme und Leistung gibt es allenfalls erste theoretische Annahmen ohne entsprechende empirische Befunde. Davis Jr. (vgl. 2001) betrachtet historisches Fachwissen als notwendige, jedoch nicht unbedingt hinreichende Voraussetzung für historische Perspektivenübernahme. Auch Ashby und Lee (vgl. 1987) betonen die Bedeutung von historischer Perspektivenübernahme für gute Leistungen im Geschichtsunterricht. Zudem weisen sie darauf hin, dass erst die histo- 
rische Perspektivenübernahme es ermögliche, von der Kenntnis historischer Fakten zum adäquaten Verständnis vergangener Handlungen zu gelangen (Lee \& Ashby 2001).

Das Zusammenspiel von Teilkompetenzen historischen Lernens mit Indikatoren für fachspezifische Leistung und Motivation ist bisher noch nicht genauer untersucht worden. Gehlbach (vgl. 2006) betrachtet Perspektivenübernahme, Interesse und Wissen in seiner Studie als abhängige Variablen, geht dabei aber nicht auf die Zusammenhänge zwischen ihnen ein.

\section{Ziel dieser Studie}

Die vorliegende Studie soll das bisher hauptsächlich theoretisch vermutete Zusammenspiel zwischen der Schülerkompetenz historische Perspektivenübernahme, fachspezifischen Merkmalen sowie der Fähigkeit zur sozialen Perspektivenübernahme erstmals empirisch beleuchten. Da bisher nur sehr wenige empirische Studien zu dieser Teilkompetenz des historischen Lernens vorliegen, wählen wir einen explorativen Zugang und betrachten Zusammenhänge zwischen fachspezifischen Leistungs- und Motivationsindikatoren, der sozial-kognitiven Fähigkeit zur sozialen Perspektivenübernahme sowie einem neu entwickelten Maß für historische Perspektivenübernahme (vgl. Hartmann \& Hasselhorn 2008; Hartmann, Martens \& Sauer 2007). Als Leistungsindikatoren verwenden wir die Geschichtsnote sowie das Abschneiden in einem Lückentext zu historischen Themen, die zuvor im Unterricht behandelt wurden. Als Motivationsindikator untersuchen wir das Interesse am Fach Geschichte. Weiterhin nehmen wir das geschichtsbezogene Selbstkonzept in unsere Untersuchung auf, welches nach Rost und Sparfeldt (vgl. 2002) sowohl Beziehungen zur Leistung als auch zum Interesse aufweisen soll. Aufseiten der sozial-kognitiven Schülermerkmale untersuchen wir sowohl die Fähigkeit von Schülern, anhand eines fiktiven Szenarios soziale Perspektiven einzunehmen, als auch ihre Bereitschaft, diese Fähigkeit im Alltag zu zeigen.

Wir vermuten erstens, dass sich die bereits aus anderen Schulfächern bekannten und auch für das Fach Geschichte vereinzelt berichteten Zusammenhänge zwischen Schulnote, Selbstkonzept und Interesse auch in unserer Studie zeigen werden. Zweitens halten wir es für plausibel, dass positive Zusammenhänge zwischen der spezifischen Teilkompetenz historische Perspektivenübernahme und fachspezifischen Leistungs- und Motivationsindikatoren bestehen. Drittens halten wir aufgrund der theoretischen Nähe der beiden Konstrukte positive Zusammenhänge zwischen sozialer und historischer Perspektivenübernahme für denkbar. In diesem noch weitgehend unbearbeiteten Forschungsfeld erscheint uns die Formulierung spezifischer gerichteter Hypothesen zum jetzigen Zeitpunkt jedoch verfrüht. Vielmehr soll diese Studie dazu dienen, einen ersten empirischen Einblick in das Zusammenspiel fachspezifischer und sozial-kognitiver Schülermerkmale sowie einer Teilkompetenz historischen Denkens zu erhalten, auf deren Basis die Formulierung prüfbarer Hypothesen erst möglich wird. 


\section{Methode}

\subsection{Stichprobe}

Die Daten der vorliegenden Studie entstammen einer Fragebogenerhebung, die an drei niedersächsischen Gymnasien durchgeführt wurde. Es wurden alle Schüler/-innen der 7. und 10. Klassen $(\mathrm{N}=375)$ befragt. 208 Personen $(110$ weiblich $)$ mit einem Altersdurchschnitt von $M=13.03$ Jahren besuchten zum Zeitpunkt der Untersuchung die 7. Klasse. 167 Personen (97 weiblich) mit einem Altersdurchschnitt von $M=16.13$ Jahren entstammten den 10. Klassen. 38\% der Stichprobe wiesen einen Migrationshintergrund auf (entweder Schüler/-in oder mindestens ein Elternteil im Ausland geboren).

\subsection{Messinstrumente}

Im Folgenden sind die in der Studie eingesetzten Messinstrumente aufgeführt, auf die sich die berichteten Ergebnisse beziehen. Tabelle 1 informiert über die Kennwerte zur internen Konsistenz der Skalen, getrennt für beide Klassenstufen.

Historische Perspektivenübernahme. Die Erfassung historischer Perspektivenübernahme erfolgte im Anschluss an die Präsentation eines lehrplanbezogenen fiktiven Szenarios, in dem eine historische Person handelt. Alle Schüler sollten sich das Szenario zunächst durchlesen. Sie wurden instruiert, sich in die handelnde Person hineinzuversetzen und ihre Zustimmung oder Ablehnung zu vierstufigen Items abzugeben, von denen jeweils drei eine gegenwartsfixierte Betrachtung der historischen Situation implizieren und drei weitere den historischen Kontext und Unterschiede zwischen Gegenwart und Vergangenheit berücksichtigen.

Tab. 1: Interne Konsistenzen der eingesetzten Messinstrumente

\begin{tabular}{llll}
\hline & Skala & $\begin{array}{l}\text { Cronbachs } \\
\text { Alpha }\end{array}$ & $\begin{array}{l}\text { Anzahl der } \\
\text { Items }\end{array}$ \\
\hline 7. Klasse & Historische PÜ (Kloster) & .51 & 6 \\
& Fähigkeit soziale Perspektivenkoordination & .85 & 9 \\
& Soziale PÜ (Selbstbericht) & .74 & 5 \\
& Interesse Geschichte & .65 & 2 \\
& Selbstkonzept Geschichte & .85 & 8 \\
& Lückentext „Mittelalter“ & .61 & 9 \\
10. Klasse $N=167)$ & Verbalskala KFT & .68 & 25 \\
& Historische PÜ (Wahl) & .78 & 6 \\
& Fähigkeit soziale Perspektivenkoordination & .85 & 9 \\
& Soziale PÜ (Selbstbericht) & .76 & 5 \\
& Interesse Geschichte & .73 & 2 \\
& Selbstkonzept Geschichte & .89 & 8 \\
& Lückentext „Weimar/Zweiter Weltkrieg“ & .76 & 13 \\
& Verbalskala KFT & .70 & 25 \\
\hline
\end{tabular}

Anmerkung: PÜ = Perspektivenübernahme. 
Das Szenario für Schüler der 7. Klasse (Kloster) ist im Mittelalter angesiedelt: Die 22jährige Johanna ist die Tochter eines Freiherrn und soll mit einem ihr weitgehend unbekannten Mann verheiratet werden, der ihrem Stand entspricht. Als diese Hochzeitspläne scheitern, weil der vorgesehene Mann sich für eine wohlhabendere und standeshöhere Frau entschieden hat, beschließen ihre Eltern, Johanna in ein Kloster zu geben, damit sie dort finanziell abgesichert leben kann. Die Schüler sollten einschätzen, was es für Johanna bedeutet, in ein Kloster geschickt zu werden. Der vollständige Text ist abgedruckt bei Hartmann, Martens und Sauer (2007).

Das Szenario für Schüler der 10. Klasse (Wahl) handelt von Hannes, dem Sohn eines bürgerlichen Schuhfabrikanten zur Zeit der Weimarer Republik: Die Firma seines Vaters steht vor dem Aus. Hannes ist verzweifelt über den Zustand seines Landes und die wirtschaftlichen und sozialen Probleme und überlegt, welcher Partei er bei der kommenden Reichstagswahl seine Stimme geben soll. Politisch steht Hannes der DNVP nahe, fragt sich aber, ob deren Führungspersönlichkeiten einflussreich genug sind, um die Krise abzuwenden. Die Schüler sollen einschätzen, ob Hannes seine Stimme einer antidemokratischen Partei wie der NSDAP geben wird. Eine ausführliche Beschreibung dieses Szenarios findet sich bei Hartmann und Hasselhorn (2008). Die Items zu beiden Szenarien sind in Tab. 2 abgebildet.

Tab. 2: Items zur Erfassung historischer Perspektivenübernahme

\begin{tabular}{lll}
\hline & 7. Klasse (Kloster-Szenario) & 10. Klasse (Wahl-Szenario) \\
\hline Gegenwarts- & (G1) Für Johanna muss es furchtbar & (G1) Die NSDAP wird er bestimmt \\
fixierung (G) & $\begin{array}{l}\text { sein, ins Kloster zu gehen, weil sie } \\
\text { dann keine Chance mehr hat, jemanden } \\
\text { kennen zu lernen, den sie liebt. }\end{array}$ & $\begin{array}{l}\text { nicht wählen. Was die mit Deutschland } \\
\text { und der Welt angerichtet haben, darf } \\
\text { man nicht zulassen. }\end{array}$ \\
& $\begin{array}{ll}\text { (G2) Sie wird ihre Eltern grausam } \\
\text { finden, weil sie sie in ein Kloster geben } \\
\text { und ihr keine Möglichkeit geben zu } \\
\text { heiraten. }\end{array}$ & $\begin{array}{l}\text { nur in einer Demokratie mitbestimmen } \\
\text { können. Darum wird er eine vernünf- } \\
\text { tige Entscheidung treffen und die heißt }\end{array}$ \\
& bestimmt nicht NSDAP.
\end{tabular}

(G3) Johanna wird sich von ihren Eltern (G3) Ich glaube nicht, dass er die schlecht behandelt fühlen, weil sie ihr nicht ermöglichen, ein eigenes, selbstbestimmtes Leben zu führen.

Berücksich- (K1) Auf die Heirat zu verzichten ist tigung des für Johanna nicht so schlimm. Für sie historischen ist vor allem wichtig, dass sie für ihr Kontextes (K) Leben versorgt ist.

(K2) Johanna weiß, dass ihre Eltern sie mit guten Absichten ins Kloster geben, damit sie auch als unverheiratete Frau versorgt ist.

NSDAP wählt. Deren Parolen sind doch leicht zu durchschauen und es ist klar, dass das auf Krieg hinausläuft.

(K1) Hannes fehlt die Erfahrung mit der Demokratie. Er weiß nicht, welches Risiko die NSDAP bedeutet und wird sie wahrscheinlich wählen.

(K2) Für ihn stellt Hitler einen starken Führer dar, der zeigt, wo es langgeht. An die Gefahren, die von der NSDAP ausgehen, denkt Hannes eher nicht.

(K3) Religion spielt für Johanna eine ganz andere Rolle als für viele Menschen heute. Deshalb ist es für sie sinnvoll, ein Leben im Kloster zu führen.

(K3) In seiner Situation sieht er nur Nachteile der Demokratie. Deshalb werden ihn die Parolen der NSDAP ansprechen. 
Zur Skalenbildung wurden die jeweils sechs Items jedes Szenarios Faktoren- und Korrelationsanalysen unterzogen. In den Daten der 10. Klasse konnte für diese Items eine klare einfaktorielle Struktur nachgewiesen werden mit den Polen Gegenwartsfixierung und Berücksichtigung des historischen Kontextes (vgl. auch Hartmann \& Hasselhorn 2008). Die beiden Itemgruppen korrelieren untereinander mit $r=-.60, p<.01$. Für die Daten der 7. Klasse lassen sich zwei Faktoren extrahieren. Dabei entfallen jedoch nur zwei Items der Gruppe Berücksichtigung des historischen Kontextes (K1 und K3) auf den zweiten Faktor. Das dritte Item dieser Gruppe (K2) lädt substanziell negativ auf dem ersten Faktor. Auch K1 und K3 weisen zwar geringe, aber negative Ladungen auf dem ersten Faktor auf. Es besteht auch in dieser Klassenstufe eine substanzielle negative Korrelation zwischen den Gruppen Gegenwartsfixierung und Berücksichtigung des historischen Kontextes $(r=-.27, p<.01)$. Aufgrund dieser offensichtlichen Beziehung zwischen den beiden Itemgruppen haben wir trotz der weniger eindeutigen Datenlage in Klasse 7 jeweils einen Skalenwert für historische Perspektivenübernahme gebildet. Die Items zur Gegenwartsfixierung sind umkodiert, sodass hohe Werte für hohe historische Perspektivenübernahmekompetenz stehen. Die interne Konsistenz ist in der 10. Klasse gut $(\alpha=.78)$ und in der 7. Klasse für die Zwecke dieser Studie noch ausreichend $(\alpha=.51)$.

Fähigkeit zur sozialen Perspektivenkoordination. Die soziale Perspektivenkoordination wurde in Anlehnung an Selman (vgl. 1980) ebenfalls mithilfe von fiktiven sozialen Situationen erfasst. Es wurde das Freundschafts-Dilemma aus dem deutschen Written Interpersonal Understanding Interview von Gutzwiller-Helfenfinger (vgl. 2003) eingesetzt. Im Original besteht dieses Instrument aus 15 offenen Fragen (z. B. „Was ist in dieser Geschichte das Problem? Warum ist das ein Problem?“), die sich an die Präsentation des Dilemmas anschließen. Für die vorliegende Studie wurden nur die ersten neun Fragen eingesetzt, da in diesen bereits alle Aspekte des Kodierschemas enthalten sind. $30 \%$ der Schülerantworten wurden durch zwei unabhängige Rater in Bezug auf das Level der Perspektivenkoordination kodiert, die eine prozentuale Übereinstimmung von 74\% erreichten (Cohens $\kappa=.53$ ). Dies ist zwar nur zufriedenstellend, entspricht jedoch den bei Gutzwiller-Helfenfinger (vgl. 2003) erzielten Werten und erscheint nach den Ausführungen von Wirtz und Caspar (vgl. 2002) noch annehmbar, da es sich bei sozialer Perspektivenübernahme um ein relativ komplexes Konstrukt sozialer Kompetenz handelt.

Soziale Perspektivenübernahme (Selbstbericht). Die Schüler wurden nach ihrer Bereitschaft gefragt, im Alltag Perspektiven anderer Personen zu übernehmen. Dazu wurde die Skala Perspective Taking (vgl. Davis 1983) in der deutschen Übersetzung eingesetzt (vgl. Kunter et al. 2002). Items wie „Bevor ich Leute kritisiere, versuche ich mir vorzustellen, wie es mir ginge, wenn ich an ihrer Stelle wäre“" wurden von den Schülern auf einer vierstufigen Skala ( 1 =trifft gar nicht $\mathrm{zu} ; 4=$ trifft völlig zu) beantwortet.

Interesse an Geschichte. Das Geschichtsinteresse wurde über zwei Items erfasst, die aus der PISA-Skalendokumentation (vgl. Kunter et al. 2002) entnommen und für das Fach Geschichte angepasst wurden. Die Items lauteten „Mir macht Geschichtsunterricht Spaß“ und „Ich lerne in Geschichte etwas, das für mich sehr wichtig ist“" und wurden ebenfalls auf einer vierstufigen Skala ( 1 =trifft gar nicht zu; $4=$ trifft völlig zu) beantwortet.

Fachspezifisches Selbstkonzept Geschichte. Die acht Items zum fachspezifischen Selbstkonzept wurden aus dem DISK-Gitter von Rost und Sparfeldt (vgl. 2002) übernommen. Aussagen wie „Ich gehöre in Geschichte zu den Guten“ wurden von den 
Schülern ebenfalls auf einer vierstufigen Skala ( $1=$ trifft gar nicht zu; $4=$ trifft völlig zu) beantwortet.

Kenntnis zentraler historischer Fakten und Zusammenhänge. In beiden Klassenstufen wurde zur Überprüfung des Vorwissens ein Lückentext eingesetzt, der sich am Thema des historischen Szenarios orientierte und die Kenntnis zentraler Fakten und Zusammenhänge erfasste. In der 7. Klasse sollten neun Begriffe zum Thema Mittelalter in die Lücken geschrieben werden, in der 10. Klasse 13 Begriffe zum Thema Weimarer Republik/Zweiter Weltkrieg.

Geschichtsnote. ${ }^{3}$ Die Schüler wurden nach ihrer letzten Zeugnisnote im Fach Geschichte gefragt. Die Antworten wurden nach dem Punktesystem für Schulnoten kodiert (0 bis 15 Punkte).

Verbale Intelligenz. Eine mögliche Artefaktquelle in dem hier gewählten Vorgehen zur Erfassung historischer Perspektivenübernahme besteht darin, dass die kompetente Bearbeitung der Szenarien allein die sprachlichen Fähigkeiten der Schüler widerspiegelt. Um dies zu kontrollieren, wurde die verbale Intelligenz als zusätzliche Variable eingesetzt und durch die Verbalskala V1 des Kognitiven Fähigkeitstests (KFT; vgl. Heller \& Perleth 2000) erfasst. Maximal waren 25 Punkte zu erreichen.

\section{Ergebnisse}

In Tab. 3 sind die Mittelwerte und Standardabweichungen der untersuchten Variablen dargestellt. Die Mittelwerte der Zehntklässler liegen bezogen auf fast alle erhobenen Variablen etwas über dem Niveau der Siebtklässler. Nur das Selbstkonzept in Geschichte und die selbstberichtete Bereitschaft zur sozialen Perspektivenübernahme weisen in beiden Klassenstufen ähnliche Durchschnittswerte auf. Die Kompetenz zur historischen Perspektivenübernahme (erhoben am Kloster-Szenario) ist in der 7. Klasse um ca. einen Skalenpunkt geringer ausgeprägt als die am Wahl-Szenario erhobene Kompetenz der Zehntklässler.

Die Angaben der SchülerInnen zur Geschichtsnote wurden vor den weiteren Analysen einer klassenspezifischen z-Transformation unterzogen, sodass in jeder befragten Schulklasse ein Mittelwert von 0 und eine Standardabweichung von 1 vorlagen. Dies diente dazu, die Schulnoten, die aufgrund unterschiedlicher sozialer Bezugsnormen in den einzelnen Klassen zustande kamen, über Schulklassen hinweg vergleichbar zu machen. Unter dieser Voraussetzung halten wir es für gerechtfertigt, die Werte aus mehreren Schulklassen in einer Analyse zu berücksichtigen.

Es wurden Korrelationen zwischen der Kompetenz zur historischen Perspektivenübernahme, den geschichtsspezifischen und den sozial-kognitiven Indikatoren sowie der Verbalskala des KFT berechnet. In Tab. 4 sind die Ergebnisse der 7. Klassenstufe aufgeführt. Die Geschichtsnote, das Interesse am Fach Geschichte und das fachspezifische Selbstkonzept hängen signifikant positiv miteinander zusammen. Interesse und Geschichtsnote korrelieren zu $r=.21$. Das Selbstkonzept korreliert zu $r=.56$ sowohl mit der Geschichtsnote als auch mit dem Interesse an Geschichte. Die Kompetenz zur historischen Perspektivenübernahme korreliert in dieser Klassenstufe hochsignifikant mit der Kompetenz zur sozialen Perspektivenübernahme $(r=.20)$. Weiterhin besteht ein signifikanter Zusam- 
Tab. 3: Deskriptive Statistiken zu den eingesetzten Skalen

\begin{tabular}{llrr}
\hline & Variable & \multicolumn{1}{c}{$S$} & \multicolumn{1}{c}{$S$} \\
\hline 7. Klasse & historische PÜ & 2.15 & .44 \\
& Geschichtsnote & 8.66 & 2.88 \\
& Interesse an Geschichte & 2.43 & .78 \\
& Selbstkonzept Geschichte & 2.53 & .56 \\
& Summe Lückentext Mittelalter & 3.64 & 1.83 \\
& KFT Untertest V1 & 15.86 & 3.55 \\
& Fähigkeit soziale Perspektivenkoordination & 2.28 & .18 \\
& Soziale PÜ (Selbstbericht) & 2.69 & .58 \\
$(N=167)$ & historische PÜ & 3.19 & .52 \\
& Geschichtsnote & 8.37 & 2.99 \\
& Interesse an Geschichte & 2.82 & .81 \\
& Selbstkonzept Geschichte & 2.53 & .65 \\
& Lückentext Weimar/Zweiter Weltkrieg & 6.53 & 3.02 \\
& KFT Untertest V1 & 16.28 & 3.68 \\
& Fähigkeit soziale Perspektivenkoordination & 2.38 & .18 \\
& Soziale PÜ (Selbstbericht) & 2.77 & .57 \\
\hline
\end{tabular}

Anmerkung: PÜ=Perspektivenübernahme.

menhang zur Bereitschaft der Schüler, im Alltag Perspektiven anderer zu übernehmen $(r=.14)$. Dieses Selbstberichtsmaß zur sozialen Perspektivenübernahme korreliert außerdem mit dem Interesse $(r=.23)$ und dem Selbstkonzept in Geschichte $(r=.22)$. Weder die fachspezifischen Motivations- und Leistungsvariablen noch der Untertest des KFT korreliert signifikant mit der historischen Perspektivenübernahme.

Tabelle 5 zeigt die Korrelationstabelle für die 10. Klassenstufe. Auch in dieser Altersgruppe hängen die fachspezifischen Leistungs- und Motivationsindikatoren positiv miteinander zusammen. Interesse und Geschichtsnote korrelieren zu $r=.50$. Das Selbstkonzept korreliert zu $r=.65$ sowohl mit der Geschichtsnote als auch mit dem Interesse an Geschichte. Die Kompetenz zur historischen Perspektivenübernahme korreliert signifikant mit allen fachspezifischen Motivations- und Leistungsvariablen, am stärksten mit dem Interesse $(r=.44)$ und dem Selbstkonzept in Geschichte $(r=.48)$. Auch zum Abschneiden im Lückentext $(r=.25)$ und zur Geschichtsnote $(r=.39)$ bestehen signifikante Zusammenhänge. Die Kompetenz zur historischen Perspektivenübernahme hängt in dieser Klassenstufe nicht mit den beiden Indikatoren sozialer Perspektivenübernahme zusammen.

\section{Zusammenfassung der Ergebnisse}

Ziel dieser explorativen Studie war es, einen ersten Einblick in das Zusammenspiel zwischen einer Teilkompetenz historischen Denkens (historische Perspektivenübernahme), fachspezifischen Motivations- und Leistungsindikatoren und der sozial-kognitiven Fähigkeit zur sozialen Perspektivenübernahme zu erhalten. 


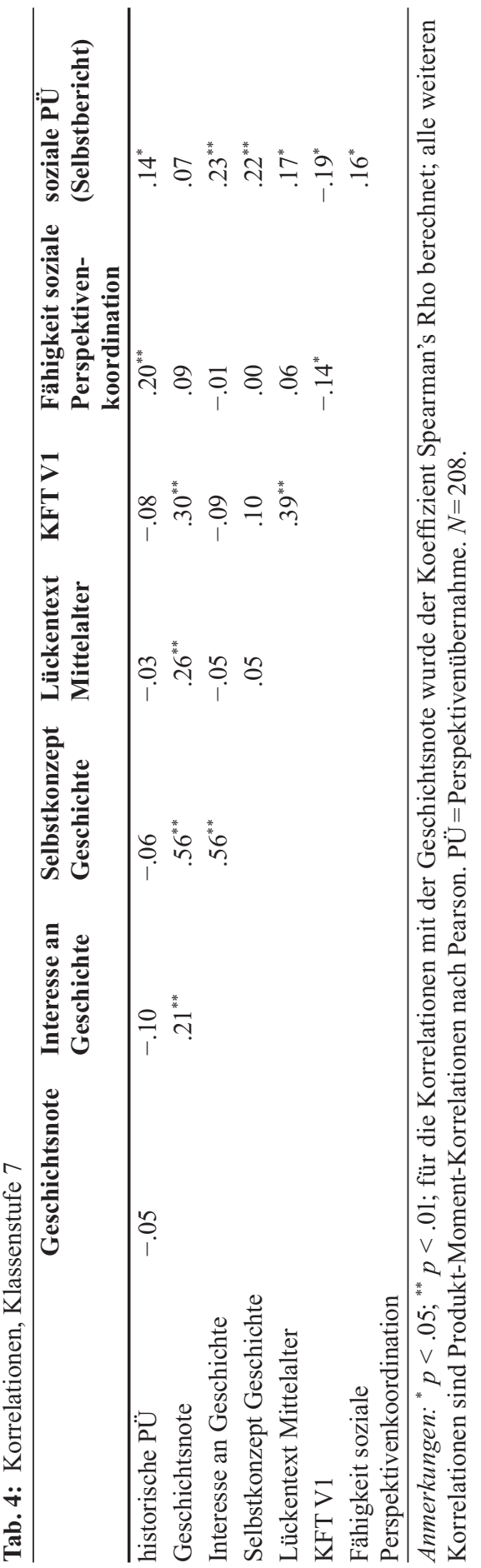




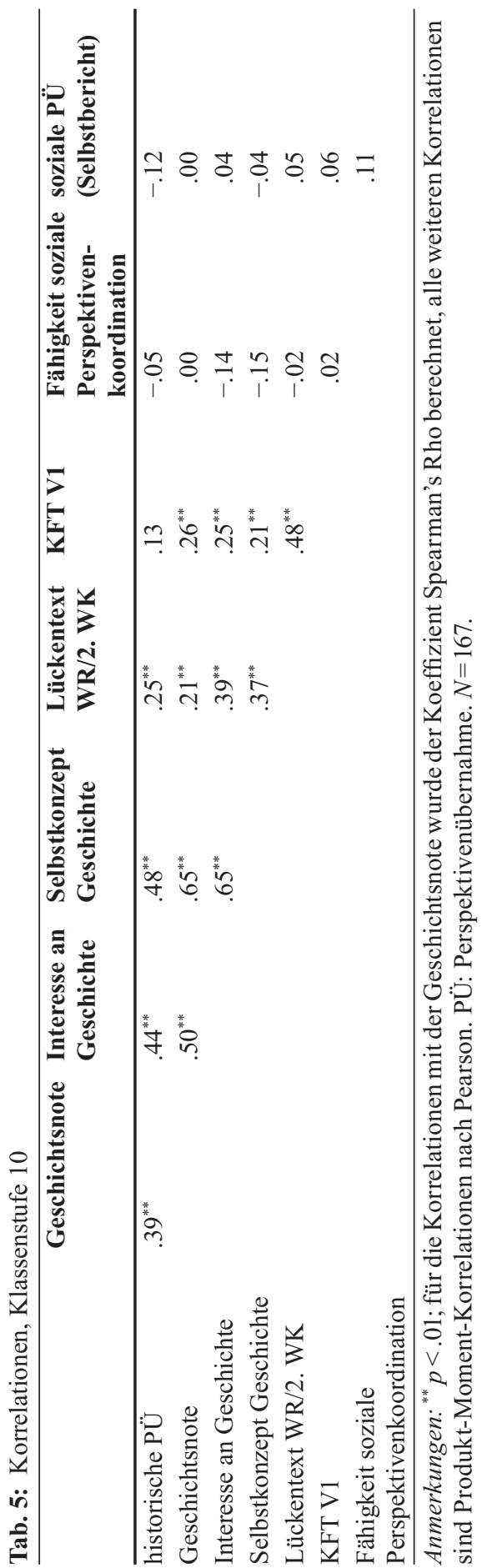


Unsere erste Vermutung war, dass sich auch im Fach Geschichte positive Zusammenhänge zwischen der Schulnote, dem fachspezifischen Interesse und dem Selbstkonzept zeigen. Diese Vermutung konnte für beide untersuchten Klassenstufen bestätigt werden und die Befunde stehen im Einklang mit den Ergebnissen von Rost und Sparfeldt (vgl. 2002). Dass die Stärke der Zusammenhänge in der 10. Klasse durchweg höher ist als in der 7. Klasse, ist außerdem konsistent mit den Daten für die Fächer Englisch, Naturwissenschaften und Mathematik aus der Längsschnittstudie von Denissen, Zarrett und Eccles (vgl. 2007). Vor allem der Zusammenhang zwischen der Geschichtsnote und dem Interesse an Geschichte ist in der 10. Klasse deutlich höher als in der 7. Klasse (.50 vs .21).

Zweitens haben wir untersucht, welche Zusammenhänge zwischen der historischen Perspektivenübernahme und den fachspezifischen Leistungs- und Motivationsindikatoren bestehen. Während wir in der 7. Klasse keinerlei substanzielle Zusammenhänge feststellen konnten, bestehen in der 10. Klasse hochsignifikante Korrelationen der historischen Perspektivenübernahme mit allen fachspezifischen Merkmalen. Insbesondere das Interesse und das Selbstkonzept korrelieren relativ hoch (.44 und .48) mit der kompetenten Bearbeitung des historischen Szenarios, aber auch die Geschichtsnote (.39) und die Beantwortung des themenspezifischen Lückentexts (.25) hängen statistisch bedeutsam mit der Kompetenz zur historischen Perspektivenübernahme zusammen.

Drittens sind wir der Frage nachgegangen, ob Zusammenhänge zwischen der Kompetenz zur historischen Perspektivenübernahme und zwei Indikatoren für das sozialkognitive Merkmal der sozialen Perspektivenübernahme bestehen. Zum einen wurde die Fähigkeit zur sozialen Perspektivenübernahme mit offenem Antwortformat in Anlehnung an Selman (vgl. 1980 sowie Gutzwiller-Helfenfinger 2003) erhoben. Zum anderen wurde die Bereitschaft, Perspektiven von Interaktionspartnern im Alltag zu übernehmen, mittels eines Selbstberichtsverfahrens erfasst. Während in der 10. Klasse keinerlei Zusammenhänge zwischen den fachspezifischen und den sozial-kognitiven Merkmalen bestehen, deuten die Befunde der 7. Klasse auf eine Verbindung zwischen historischer und sozialer Perspektivenübernahme hin. Die Korrelationen liegen im niedrigen bis moderaten Bereich - ein Befund, der sich mit den Ergebnissen Gehlbachs (vgl. 2004a) deckt. Die Bereitschaft, Perspektiven anderer Personen im Alltag einzunehmen, hängt in der 7. Klasse außerdem mit dem Interesse und Selbstkonzept im Fach Geschichte zusammen.

Da diese pädagogisch und entwicklungspsychologisch interessanten Ergebnisse innerhalb einer explorativen Querschnittsstudie zustande gekommen sind und auf korrelativen Daten basieren, sind daraus nur begrenzt Schlussfolgerungen über die Entwicklung bzw. den Erwerb der Kompetenz zur historischen Perspektivenübernahme möglich. Um unsere Ergebnisse für die Theoriebildung und weitere empirische Untersuchungen nutzbar zu machen, gehen wir im folgenden Abschnitt auf mögliche Wirkzusammenhänge ein, im Bewusstsein, dass diese Vermutungen aufgrund der vielfältigen Grenzen der Studie zum jetzigen Zeitpunkt noch sehr spekulativ bleiben müssen.

Zuvor möchten wir noch einen Randbefund dieser Studie erwähnen, der auf den ersten Blick verwunderlich erscheint. Die Fähigkeit zur sozialen Perspektivenübernahme korreliert in unseren Daten nur gering (in der 7. Klasse) bis gar nicht (in der 10. Klasse) mit der Bereitschaft zur historischen Perspektivenübernahme. Dies kann mehrere Gründe haben: Zum einen muss auf einen möglichen Methodeneffekt aufgrund der unterschiedlichen Erhebungsmethoden hingewiesen werden (offenes vs. geschlossenes Antwortfor- 
mat). Zum anderen weist Steins (vgl. 1998) darauf hin, dass Selbstberichtsverfahren zur Perspektivenübernahme und Empathie einer starken Tendenz zur sozialen Erwünschtheit unterliegen können.

\section{Mögliche Wirkzusammenhänge sozial-kognitiver und geschichtsspezifischer Schülermerkmale}

Unsere Studie wirft zahlreiche Fragen auf, die das Wirkungsgefüge zwischen fachspezifischen und sozial-kognitiven Schülermerkmalen bezogen auf das Fach Geschichte betreffen. Ausgehend von den Daten der 7. Klasse sollte zukünftig untersucht werden, ob die Kompetenz zur historischen Perspektivenübernahme bei Schülern zunächst auf deren sozial-kognitive Fähigkeit und Bereitschaft zurückzuführen ist, im Alltag fremde Perspektiven einzunehmen. Naheliegend ist außerdem die Frage, ob eine solche sozialkognitive Kompetenz das Interesse an Fächern wie Geschichte fördern kann. Die von uns gefundene Korrelation zwischen der Bereitschaft zur Perspektivenübernahme und dem Geschichtsinteresse stellt dafür eine erste notwendige, wenngleich noch keinesfalls hinreichende Bedingung dar.

Die Daten der 10. Klasse regen zu weiteren Fragen und Vermutungen an: Historische Perspektivenübernahme reiht sich in dieser Klassenstufe in das Beziehungsgefüge der fachspezifischen Leistungs- und Motivationsindikatoren ein. Die Fähigkeit und Bereitschaft zur sozialen Perspektivenübernahme hängt mit diesen fachspezifischen Merkmalen nicht mehr zusammen. Dieser Befund sollte in zukünftigen Studien überprüft werden und mit spezifischen Merkmalen des Unterrichts in Verbindung gebracht werden. Es scheint zwar im Zuge einer höheren fachlichen Spezialisierung plausibel, aber aus fachdidaktischer Sicht wenig wünschenswert, dass allgemeine sozial-kognitive Fähigkeiten im Fach Geschichte in höheren Jahrgängen eine geringere Rolle spielen - vor allem da Studien wie die von Hickey Schultz, Barr und Selman (vgl. 2001) auf die Potentiale des Faches Geschichte für den Lebensweltbezug der Schüler hinweisen.

Bezogen auf die Unterschiede zwischen den beiden Klassenstufen lassen sich entwicklungspsychologisch relevante Fragen anschließen: Stellt die sozial-kognitive Fähigkeit zur sozialen Perspektivenübernahme bei Kindern eine Voraussetzung für das Sich-Hineinversetzen in historische Situationen dar? Führt eine egozentrische Sichtweise dazu, dass historische Situationen ausschließlich nach den eigenen Wertmaßstäben der Gegenwart beurteilt werden können? Und welche Prozesse sind für eine mögliche Entwicklung dieser Kompetenzen verantwortlich? Wie sollte Geschichtsunterricht gestaltet werden, um eine aufeinander abgestimmte Förderung sozialer und fachspezifischer Kompetenzen zu ermöglichen?

Diese Fragen und Vermutungen, die sich aufgrund der empirischen Befunde ergeben, können zur weiteren Auseinandersetzung mit dem komplexen Wirkungsgefüge im Fach Geschichte anregen, müssen aber noch als sehr vorläufig betrachtet werden. Das ist zum einen im spezifischen Aufbau unserer explorativen Querschnittsstudie begründet. Zum anderen weisen die Grenzen unserer Studie aber auch auf allgemeine Herausforderungen für die empirische Kompetenzforschung in bisher wenig untersuchten Schulfächern und an der Schnittstelle zwischen Erziehungswissenschaft, Fachdidaktik und Psychologie hin. 


\section{Grenzen der Studie}

Validität des Konstruktes. Historische Perspektivenübernahme wird in der geschichtsdidaktischen Diskussion sehr breit gefasst. Schon durch die vielfältigen Begrifflichkeiten - z. B. historical empathy bei Ashby und Lee (vgl. 1987); Perspektivenübernahme oder Wahrnehmung von Perspektivität bei Sauer (vgl. 2006); Welt- und Fremdverstehen bei Körber, Schreiber und Schöner (vgl. 2007) - wird deutlich, dass das Konstrukt noch nicht sehr eng umrissen ist. Auch bezüglich der Bedeutung von historischem Wissen für diese Kompetenz gibt es noch keine einheitliche Auffassung, auch wenn Davis Jr. (vgl. 2001) sowie Ashby und Lee (vgl. 1987) die Relevanz von Kontextwissen für historische Perspektivenübernahme betonen. In unserer Studie haben wir versucht, einen ersten empirischen Zugang mithilfe eines historischen Kontextes zu schaffen, zu dem Schüler bereits durch den Unterricht vermitteltes Wissen haben sollten. Unsere Befunde sprechen dafür, dass historische Perspektivenübernahme mehr ist als reines historisches Kontextwissen. Zwar gibt es in Klasse 10 eine signifikante Korrelation mit der Kenntnis zentraler Fakten zu dem Themengebiet des Szenarios; die Größenordnung von $r=.25$ lässt jedoch die Interpretation zu, dass historische Perspektivenübernahme nicht ausschließlich vom historischen Wissen der Schüler determiniert wird.

Bei der Messung der Kompetenz haben wir uns in dieser Studie auf zwei Aspekte beschränkt, die von diversen Autoren (vgl. z. B. Ashby \& Lee 1987; Bergmann 2000; von Borries 1995; Sauer 2006; Seixas 1996) als zentral für historische Perspektivenübernahme angesehen werden - die Gegenwartsfixierung, die überwunden werden soll, und die Berücksichtigung des historischen Kontextes beim Verstehen der Akteure, die als Ziel der Entwicklung dieser Kompetenz gilt. Offen bleibt, ob es weitere zentrale Bestandteile von historischer Perspektivenübernahme gibt, die mit unserem Instrument nicht erfasst wurden. Es sind weitere Explikationen des Konstruktes notwendig, die sich unseres Erachtens sowohl an fachspezifischen als auch an pädagogisch-psychologischen Theorien zur Perspektivenübernahme orientieren sollten. Auch weitere qualitative Studien im Sinne von Ashby und Lee (vgl. 1987) können dazu beitragen, Bestandteile historischer Perspektivenübernahme im Denken von Schülern aufzudecken.

Darüber hinaus ist im Hinblick auf die Validität diskussionswürdig, ob die für die soziale Perspektivenübernahme vorgeschlagene Unterteilung zwischen Fähigkeit und Bereitschaft (vgl. Gehlbach 2004b) auch für das Konzept der historischen Perspektivenübernahme nützlich sein kann. In Weinerts Konzeption (vgl. 2001) von Kompetenz fließen sowohl Fähigkeiten als auch Bereitschaften ein, bei der konkreten Umsetzung in Kompetenzaufgaben kann man diesem Anspruch jedoch nur schwer gerecht werden. Letztlich muss jedes Testergebnis als ein Ausdruck des Zusammenspiels von Fähigkeiten und Bereitschaften angesehen werden.

Aufgabenformate. In der vorliegenden Studie wurde historische Perspektivenübernahme bei Schülern über die Präsentation eines Szenarios und die anschließende Beantwortung von Items mithilfe von Ratingskalen gemessen. Dies stellt insofern eine Ausnahme dar, als die meisten geschichtsdidaktischen Studien qualitative Studiendesigns unter Verwendung offener Antwortformate nutzen (vgl. z. B. Ashby \& Lee 1987; Shemilt 1987; Voss \& Wiley 1997; Wineburg 1991). Um Erkenntnisse über die Prozesse historischen Denkens zu sammeln, scheint dies der geeignete Weg zu sein. Insbesondere im Kontext 
von Bildungsstandards ist es jedoch notwendig, auch die Chancen und Grenzen quantitativer Studien auszuloten, da eine ökonomische Messung von Kompetenzen in größeren Schülerpopulationen erst dadurch möglich wird. Über spezifische Messprobleme, die mit der Natur historischer Denkprozesse in Verbindung stehen, sind weitere Diskussionen notwendig. Beispielsweise verweist von Borries (vgl. 2007) darauf, dass es im Bereich des historischen Denkens oftmals nicht die eine richtige Antwort geben kann. Vielmehr kommt es auf historisch plausible Argumentationen und Begründungen an, die Schüler entwickeln sollen, und die zu durchaus unterschiedlichen Interpretationen historischer Ereignisse führen könnten. Unserer Ansicht nach kann die Verwendung von Ratingskalen einen Kompromiss darstellen, da sie nicht eine einzig richtige Antwort impliziert, sondern jedes Item abgestuft und unabhängig von den anderen Items bewertet werden kann. Somit bleibt Schülern die Möglichkeit, vermeintlich gegensätzliche Aussagen in derselben Art und Weise zu bewerten, falls dies ihren historischen Konzepten entsprechen sollte. Auch wenn dies ein Problem für eine einfache Skalenbildung darstellt, eröffnet es Wissenschaftlern doch die Chance, Widersprüche und Misskonzepte zu erkennen und neue Erkenntnisse zu gewinnen. Bezogen auf die Prozessanalyse historischen Denkens kann jedoch auch die Verwendung von Ratingskalen offene Antwortformate und Methoden des lauten Denkens (vgl. Wineburg 1991) nicht ersetzen.

$\mathrm{Zu}$ diskutieren ist weiterhin die Verwendung fiktiver Szenarien, da es im Schulfach Geschichte darum geht, das Verständnis realer historischer Begebenheiten bei Schülern zu fördern. Solche eigens für den Zweck der Studie konstruierten Aufgaben haben den Vorteil, dass weniger Störfaktoren in die Messung hineinspielen, die bei der Verwendung authentischer Quellen und Darstellungen wirksam sind. Dies betrifft beispielsweise die sprachliche Komplexität der untersuchten Dokumente und die Vermischung von verschiedenen Teilprozessen historischen Denkens (z. B. das Ineinandergreifen von Quelleninterpretation und historischer Perspektivenübernahme). Dass man mit der Verwendung fiktiver Szenarien jedoch unter Umständen Einbußen in Bezug auf die Inhaltsvalidität in Kauf nimmt, soll an dieser Stelle nicht unerwähnt bleiben.

Reliabilität. Weiterhin muss auf die zum Teil geringen internen Konsistenzen vor allem bei der Erfassung historischer Perspektivenübernahme in der 7. Klasse eingegangen werden. Vermutlich setzt es bereits ein gewisses Maß an historischer Perspektivenübernahme voraus zu erkennen, ob die entsprechenden Aussagen gegenwartsfixiert sind oder den historischen Kontext einbeziehen. Dies gelingt in Klasse 7 vermutlich weniger gut als in Klasse 10, was zu einer geringeren internen Konsistenz der Skala in dieser Altersgruppe führen könnte. Um dieses Problem zu lösen, müssten die Items für Klasse 7 überarbeitet werden. Wir vermuten allerdings, dass die Zusammenhänge zu den fachspezifischen Variablen sich auch bei einer Erhöhung der Reliabilität nicht grundlegend verändern würden - die Korrelationen mit den fachspezifischen Variablen, die durchgehend um Null liegen, legen diese Interpretation nah.

$\mathrm{Zu}$ diskutieren bleibt, ob der Maßstab einer hohen internen Konsistenz, wie er in der Psychologie in der Regel angelegt wird, für die Erforschung historischen Lernens ebenso rigoros vorauszusetzen ist. Es ist fraglich, ob es der Breite der Konstrukte, die den geschichtsdidaktischen Diskurs bestimmen, gerecht wird, wenn ausschließlich die interne Konsistenz von Skalen berücksichtigt wird. Insbesondere bei den ersten Studien in diesem Bereich, die sich auf die zum Teil noch recht vage formulierten Kompetenzmo- 
delle stützen und sich an heterogenen Kompetenzbereichen orientieren müssen, sollten geringere interne Konsistenzen zwar kritisch reflektiert werden, jedoch nicht automatisch zur Eindämmung der Forschungsaktivitäten im Fach führen. Denn auch mit heterogenen Konstrukten und ersten empirischen Ansätzen ist eine Annäherung an interessante inhaltliche Fragestellungen möglich.

Kontextgebundenheit. Durch die Anlage der Studie, die historische Perspektivenübernahme in den Klassen 7 und 10 an zwei unterschiedlichen historischen Kontexten erfasst, muss der Mangel an Vergleichbarkeit der Aufgaben über die Klassenstufen hinweg diskutiert werden. Auch wenn bei der Konstruktion der Texte darauf geachtet wurde, Charakteristika wie Textlänge, Schwierigkeit und Formulierung der Items für beide Klassenstufen möglichst einheitlich zu gestalten, bleiben die historischen Kontexte unterschiedlich. Wir haben uns in dieser Studie dafür entschieden, unterschiedliche Kontexte für die beiden Klassenstufen zu wählen, damit die Schüler ihre Kompetenz zur historischen Perspektivenübernahme an zuvor im Unterricht behandelten historischen Themen zeigen konnten. Wir vermuten, dass historische Kontextualisierung (unser Anspruch an kompetente Schüler) ein Mindestmaß an Kenntnissen über den spezifischen historischen Kontext voraussetzt (vgl. z. B. Lee \& Ashby 2001; Davis Jr. 2001). Es wäre demnach nicht möglich, historische Perspektivenübernahme kontextunabhängig oder an gänzlich unbekannten Kontexten zu erfassen. Die Befunde von Beck und McKeown (vgl. 1994) lassen es zudem zweifelhaft erscheinen, ob sich Zehntklässler noch an Inhalte aus dem Geschichtsunterricht der siebten Klasse erinnern können. Andererseits sollen Kompetenzen im Sinne der Expertise von Klieme et al. (vgl. 2003) aber nicht nur an einem spezifischen Kontext bei Schülern nachgewiesen werden, sondern sich an variablen Kontexten manifestieren und sich als transferfähig herausstellen. Diese Herausforderungen in Bezug auf die Kontextgebundenheit historischer Kompetenzen sind weiter zu diskutieren, will man Kompetenzmessungen im Fach Geschichte an Schülern unterschiedlicher Altersstufen mit unterschiedlichen Unterrichtsinhalten durchführen.

Kompetenzstufen historischen Denkens? Mit der vorliegenden Studie haben wir das Konzept historischer Perspektivenübernahme durch zwei Aspekte definiert, die in der Literatur weitgehend übereinstimmend als wichtig angesehen werden (vgl. z. B. Ashby \& Lee 1987; Bergmann 2000; von Borries 1995; Sauer 2006; Seixas 1996). Dies lässt viele theoretische und methodische Fragen offen, beispielsweise ob Schüler immer mit einer gegenwartsfixierten Betrachtung historischer Kontexte beginnen, ob mit historischer Kontextualisierung, so wie sie von uns operationalisiert wurde, bereits die höchste Kompetenzausprägung erreicht ist und auf welchen Wegen Schüler von der Gegenwartsfixierung zu einer kontextualisierten Betrachtung historischer Situationen gelangen. Diese Fragen berühren die Forderung nach der Stufung von Kompetenzen (vgl. Klieme et al. 2003). Unser Beitrag liefert ausschließlich einen Vorschlag für zwei „Endpunkte“ der Kompetenz zur historischen Perspektivenübernahme. Sowohl für die Unterteilung quantitativ definierbarer Kompetenzstufen (in Form von erreichten Punktzahlen in Lernstandserhebungen) als auch für die Ableitung qualitativer Entwicklungsstufen (z.B. mit der Möglichkeit von Entwicklungssprüngen und der Aufhebung von Fehlkonzepten) reichen die bisher gesammelten Erkenntnisse in diesem Feld in keiner Weise aus. Dies gilt nicht nur für die Kompetenz, historische Perspektiven einzunehmen, sondern vermutlich für die meisten Teilbereiche historischen Denkens. 
Neben all diesen Herausforderungen, mit denen empirisch arbeitende Forscher im Fach Geschichte konfrontiert sind, sollten die damit verbundenen Chancen nicht unterschätzt werden. Denn erst durch die Sammlung empirischer Daten und die Formulierung exakterer Hypothesen können wir erkennen, welche fachspezifischen und sozialen Kompetenzen durch die Beschäftigung mit der Vergangenheit bei Schülern gefördert werden können. Dies kann letztlich zur besseren gesellschaftlichen Legitimation des Unterrichtsfaches Geschichte beitragen.

\section{Anmerkungen}

1 Diese Studie wurde durch ein Stipendium der Erstautorin im Rahmen des DFG-Graduiertenkollegs 1195 „Passungsverhältnisse schulischen Lernens“ ermöglicht.

2 Lee und Ashby und einige weitere, vornehmlich britische Forscher verwenden statt des Begriffs historische Perspektivenübernahme die Bezeichnung historical empathy. Sie beschreiben damit jedoch ebenfalls ein vornehmlich kognitives Konstrukt. Da der Empathiebegriff im Deutschen eher als eine emotionale Fähigkeit verstanden wird, haben wir uns dazu entschieden, durchgehend den Begriff historische Perspektivenübernahme zu verwenden, auch in Übereinstimmung mit Davis Jr. (vgl. 2001). Zur Diskussion um die Begriffsvielfalt siehe auch Baring (vgl. 2004).

3 Die Teilstichprobe der 10. Klasse ist weitgehend identisch mit der bei Hartmann und Hasselhorn (2008). Dort wurde bereits ein Zusammenhang zwischen historischer Perspektivenübernahme und der Geschichtsnote berichtet. Die Berechnung der sozialen Perspektivenübernahme beruhte bei Hartmann und Hasselhorn jedoch auf der latenten Klassenanalyse (LCA), in die zusätzlich ein weiterer Aspekt - die Rolle des historisch Handelnden - einfloss. In der vorliegenden Studie wird die Geschichtsnote vor dem Hintergrund der aktuellen Fragestellungen erneut in die Analyse dieser Daten mit aufgenommen, da sie als Indikator für Schulerfolg neben den in dieser Studie erstmals analysierten Variablen Interesse, Selbstkonzept und dem Abschneiden im Lückentext eine wichtige Rolle spielt.

\section{Literatur}

Ashby, R. \& Lee, P. (1987). Children's concepts of empathy and understanding in history. In C. Portal (Ed.), The history curriculum for teachers (pp. 62-88). London: Falmer.

Baring, F. (2004). Internationale geschichtsdidaktische Perspektiven - Multiperspektivität, Empathie und Perspektivenübernahme in den USA und Großbritannien. In S. Handro \& B. Schönemann (Hrsg.), Geschichtsdidaktische Lehrplanforschung: Methoden-Analysen-Perspektiven (S. 203-214). Münster: LIT Verlag.

Beck, I. L. \& McKeown, M. G. (1994). Outcomes of history instruction: Paste-up accounts. In M. Carretero \& J. F. Voss (Eds.), Cognitive and instructional processes in history and the social sciences (pp. 237-256). Hillsdale, NJ: Lawrence Erlbaum.

Bergmann, K. (2000). Multiperspektivität. Geschichte selber denken. Schwalbach/Ts.: Wochenschau-Verlag.

Bernhardt, M. (2007). Die Subjektseite der visuellen Begegnung. Vom Nutzen qualitativer empirischer Untersuchungen für die Entwicklung fachspezifischer Kompetenzen. Zeitschrift für Geschichtsdidaktik, 6, 108-124. 
Borries, B. von (1995). Das Geschichtsbewusstsein Jugendlicher. Eine repräsentative Untersuchung über Vergangenheitsdeutungen, Gegenwartswahrnehmungen und Zukunftserwartungen von Schülerinnen und Schülern in Ost- und Westdeutschland. Weinheim: Juventa.

Borries, B. von (2007). Empirie: Ergebnisse messen (Lerndiagnose im Fach Geschichte). In A. Körber, W. Schreiber \& A. Schöner (Hrsg.), Kompetenzen historischen Denkens. Ein Strukturmodell als Beitrag zur Kompetenzorientierung im Fach Geschichte (S. 653-673). Neuried: ars una.

Caldarella, P. \& Merell, K. W. (1997). Common dimensions of social skills of children and adolescents: A taxonomy of positive behaviours. School Psychology Review, 26, 264-278.

Davis, M. H. (1983). Measuring individual differences in empathy: Evidence for a multidimensional approach. Journal of Personality and Social Psychology, 44, 113-126.

Davis Jr., O. L. (2001). In pursuit of historical empathy. In O. L. Davis Jr., E. A. Yeager \& S. J. Foster (Eds.), Historical empathy and perspective taking in the social studies (pp. 1-12). Lanham, MD: Rowman \& Littlefield Publishers.

Davis Jr., O. L., Yeager, E. A. \& Foster, S. J. (Eds.) (2001). Historical empathy and perspective taking in the social studies. Lanham, MD: Rowman \& Littlefield Publishers.

Denissen, J. J. A., Zarrett, N. R. \& Eccles, J. S. (2007). I like to do it, I'm able, and I know I am: Longitudinal couplings between domain-specific achievement, self-concept, and interest. Child Development, 78, 430-447.

Gautschi, P., Moser, D. V., Reusser, K. \& Wiher, P. (Hrsg.) (2007). Geschichtsunterricht heute. Eine empirische Analyse ausgewählter Aspekte. Bern: hep Verlag.

Gehlbach, H. (2004a). Social perspective taking: A facilitating aptitude for conflict resolution, historical empathy, and social studies achievement. Theory and Research in Social Education, $32,39-55$.

Gehlbach, H. (2004b). A new perspective on perspective taking: A multidimensional approach to conceptualizing an aptitude. Educational Psychology Review, 16, 207-234.

Gehlbach, H. (2006). How changes in students' goal orientations relate to outcomes in social studies. The Journal of Educational Research, 99, 358-370.

Gutzwiller-Helfenfinger, E. (2003). Assessing social perspective-taking in adolescence: The written interpersonal understanding interview. Dissertation, Universität Bern. Zugriff am 04.02.2009 http://www.stub.unibe.ch/download/eldiss/03gutzwiller_e.pdf

Hartmann, U. \& Hasselhorn, M. (2008). Historical perspective taking - a standardized measure for an aspect of students' historical thinking. Learning and Individual Differences, 18, 264-270.

Hartmann, U., Martens, M. \& Sauer, M. (2007). Von Kompetenzmodellen zur empirischen Erforschung von Schülerkompetenzen - das Beispiel historische Perspektivenübernahme. Zeitschrift für Geschichtsdidaktik, 6, 125-148.

Heller, K. A. \& Perleth, C. (2000). Kognitiver Fähigkeits-Test (KFT 4-12+R) (3. Aufl.). Göttingen: Hogrefe.

Hickey Schultz, L., Barr, D. J. \& Selman, R. L. (2001). The value of a developmental approach to evaluating character development programmes: an outcome study of Facing History and Ourselves. Journal of Moral Education, 30, 3-27.

Jerusalem, M. \& Klein-Heßling, J. (2002). Soziale Kompetenz. Entwicklungstrends und Förderung in der Schule. Zeitschrift für Psychologie, 210, 164-174.

Johnson, D. W. (1975). Cooperativeness and social perspective taking. Journal of Personality and Social Psychology, 31, 241-244.

Klieme, E., Avenarius, H., Blum, W., Döbrich, P., Gruber, H., Prenzel, M., Reiss, K., Riquarts, K., Rost, J., Tenorth, H.-E. \& Vollmer, H. J. (2003). Expertise zur Entwicklung nationaler Bildungsstandards. Berlin: BMBF.

Kölbl, C., Tiedemann, J. \& Billmann-Mahecha, E. (2006). Die Bedeutung der Lesekompetenz für Sachfächer. Psychologie in Erziehung und Unterricht, 53, 201-212. 
Körber, A., Schreiber, W. \& Schöner, A. (Hrsg.) (2007). Kompetenzen historischen Denkens. Ein Strukturmodell als Beitrag zur Kompetenzorientierung in der Geschichtsdidaktik. Neuried: ars una.

Kunter, M., Schümer, G., Artelt, C., Baumert, J., Klieme, E., Neubrand, M., Prenzel, M., Schiefele, U., Schneider, W., Stanat, P., Tillmann, K.-J. \& Weiß, M. (2002). PISA 2000: Dokumentation der Erhebungsinstrumente (Materialien aus der Bildungsforschung, Bd. 72). Berlin: MaxPlanck-Institut für Bildungsforschung.

Lee, P. \& Ashby, R. (2001). Empathy, perspective taking, and rational understanding. In O. L. Davis Jr., E. A. Yeager \& S. J. Foster (Eds.), Historical empathy and perspective taking in the social studies (pp. 21-50). Lanham, MD: Rowman \& Littlefield Publishers.

Martens, M. (2008). Geschichtsunterricht als Ort historischen Lernens: Wie gehen Schülerinnen und Schüler mit Darstellungen von Vergangenheit um? In S. Handro \& B. Schönemann (Hrsg.), Orte historischen Lernens (S. 61-73). Münster: LIT Verlag.

National Center for History in the Schools (1996). National standards for history. Basic edition. Los Angeles, CA: National Center for History in the Schools.

Pandel, H.-J. (1987). Dimensionen des Geschichtsbewusstseins. Ein Versuch, seine Struktur für Empirie und Pragmatik diskutierbar zu machen. Geschichtsdidaktik, 12, 130-142.

Pandel, H.-J. (2005). Geschichtsunterricht nach PISA. Kompetenzen, Bildungsstandards und Kerncurricula. Schwalbach/Ts.: Wochenschau-Verlag.

Portal, C. (1987). Empathy as an objective for history teaching. In C. Portal. (Ed.), The history curriculum for teachers (pp. 89-99). London: Falmer.

Rost, D. H. \& Sparfeldt, J. R. (2002). Facetten des schulischen Selbstkonzepts. Ein Verfahren zur Messung des differentiellen Selbstkonzepts schulischer Leistungen und Fähigkeiten (DISKGitter). Diagnostica, 48, 130-140.

Sauer, M. (2002). Methodenkompetenz als Schlüsselqualifikation. Eine neue Grundlegung des Geschichtsunterrichts? Geschichte, Politik und ihre Didaktik, 30, 183-192.

Sauer, M. (2006). Kompetenzen für den Geschichtsunterricht - ein pragmatisches Modell als Basis für die Bildungsstandards des Verbandes der Geschichtslehrer. Informationen für den Geschichts- und Gemeinschaftskundelehrer, 72, 7-20.

Schreiber, W. (1999). Die Entwicklung historischer Sinnbildungskompetenzen als Ziel des historischen Lernens mit Grundschülern. In W. Schreiber (Hrsg.), Erste Begegnungen mit Geschichte. Grundlagen historischen Lernens (S. 15-76). Neuried: ars una.

Schreiber, W. (2008). Ein Kompetenz-Strukturmodell historischen Denkens. Zeitschrift für Pädagogik, 54, 198-212.

Seixas, P. (1996). Conceptualizing the growth of historical understanding. In D. Olsen \& N. Torrance. (Eds.), Handbook of education and human development: New models of learning, teaching, and schooling (pp. 765-783). Oxford: Blackwell.

Selman, R. L. (1980). The growth of interpersonal understanding. Developmental and clinical analyses. New York: Academic Press.

Selman, R. L. (2003). The promotion of social awareness. Powerful lessons from the partnership of developmental theory and classroom practice. New York: Russell Sage Foundation.

Shemilt, D. (1987). Adolescent ideas about evidence and methodology in history. In C. Portal (Ed.), The history curriculum for teachers (pp. 39-61). London: Falmer.

Steins, G. (1998). Diagnostik von Empathie und Perspektivenübernahme: Eine Überprüfung des Zusammenhangs beider Konstrukte und Implikationen für die Messung. Diagnostica, 44, $117-129$.

Stodolsky, S. S., Salk, S. \& Glaessner, B. (1991). Student views about learning math and social studies. American Educational Research Journal, 28, 89-116.

Tenorth, H.-E. (2008). Bildungsstandards außerhalb der Kernfächer. Herausforderungen für den Unterricht und die fachdidaktische Forschung - Zur Einleitung in den Thementeil. Zeitschrift für Pädagogik, 54, 159-162. 
Verband der Geschichtslehrer Deutschlands e.V. (2006). Bildungsstandards Geschichte. Rahmenmodell Gymnasium 5.-10. Jahrgangsstufe. Zugriff am 04.02.2009 http://www.geschichtslehrerverband.org/fileadmin/images/pdf/bildungsstandards.pdf

Voss, J. F. \& Wiley, J. (1997). Conceptual understanding in history. European Journal of Psychology of Education, 12, 147-158.

Weinert, F. E. (2001). Vergleichende Leistungsmessung in Schulen - eine umstrittene Selbstverständlichkeit. In F. E. Weinert (Hrsg.), Leistungsmessungen in Schulen (S. 17-31). Weinheim: Beltz.

Wineburg, S. (1991). Historical problem solving: A study of the cognitive processes used in the evaluation of documentary and pictorial evidence. Journal of Educational Psychology, 83, 73-87.

Wineburg, S. (1999). Historical thinking and other unnatural acts. Phi Delta Kappan, 80, 488-499.

Wirtz, M. \& Caspar, F. (2002). Beurteilerübereinstimmung und Beurteilerreliabilität. Göttingen: Hogrefe.

Wolters, C. A. \& Pintrich, P. R. (1998). Contextual differences in student motivation and self-regulated learning in mathematics, english, and social studies classrooms. Instructional Science, 26, 27-47.

Yeager, E. A. \& Foster, S. J. (2001). The role of empathy in the development of historical understanding. In O. L. Davis Jr., E. A. Yeager \& S. J. Foster (Eds.), Historical empathy and perspective taking in the social studies (pp. 13-20). Lanham, MD: Rowman \& Littlefield Publishers.

Yeates, K. O. \& Selman, R. L. (1989). Social competence in the schools: Toward an integrative developmental model for intervention. Developmental Review, 9, 64-100. 